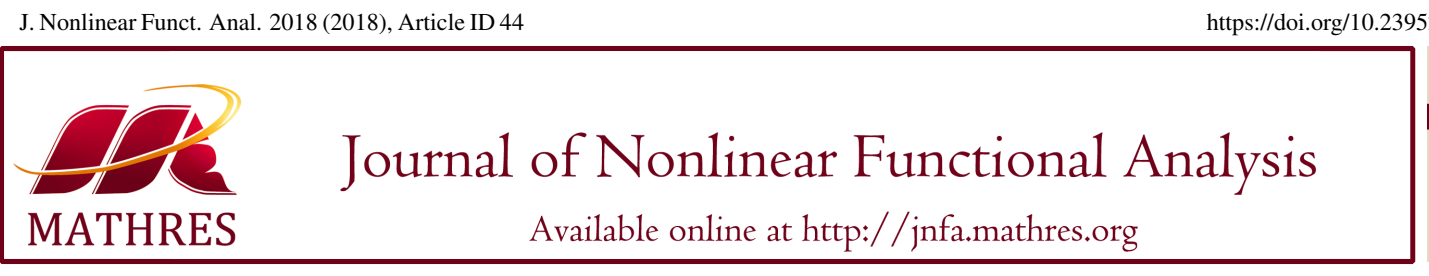

\title{
STABILITY FOR IMPULSIVE FRACTIONAL DIFFERENTIAL INCLUSIONS VIA PICARD OPERATORS IN BANACH SPACES
}

\author{
SAID ABBAS ${ }^{1}$, MOUFFAK BENCHOHRA $^{2, *}$, NAIMA HAMIDI ${ }^{2}$ \\ ${ }^{1}$ Laboratory of Mathematics, Geometry, Analysis, Control and Applications, \\ Tahar Moulay University of Saïda, P.O. Box 138, EN-Nasr, 20000 Saïda, Algeria \\ ${ }^{2}$ Laboratory of Mathematics, Djillali Liabes University of Sidi Bel-Abbès, P.O. Box 89, Sidi Bel-Abbès 22000, Algeria
}

\begin{abstract}
In this paper, we investigate the existence of mild solutions and the Ulam stability of these solutions for a class of semilinear fractional differential inclusions with not instantaneous impulses in separable Banach spaces. These results are achieved based on Picard operators and the semigroup theory. An illustrative example is also presented.

Keywords. Abstract fractional differential inclusion; Left-sided mixed Riemann-Liouville integral; Caputo fractional order derivative; Mild solution; Impulse.
\end{abstract}

2010 Mathematics Subject Classification. 26A33, 34A37, 34A6.

\section{INTRODUCTION}

In applied mathematics, the fractional calculus theory is a powerful tool to study a number of problems in science and engineering. Such problems appear in thermodynamics, finance, astrophysics, bioengineering, hydrology, mathematical physics, biophysics, statistical mechanics, theory of control and cosmology. Recently, fractional ordinary and partial differential equations, and ordinary and partial differential inclusions of fractional orders have been extensively investigated by many authors; see Abbas and Benchohra [1, 2], Abbas, Benchohra and Nieto [3], Diethelm and Ford [4], Wang and Zhang [5] and Xiang, Zhang and Wei [6] and the references therein. Recently the theory of Picard operators was introduced to study fixed point problems, see [7, 8, 9] and references therein. Subsequently, many mathematicians used this abstract approach and it seemed to be a very powerful and useful method in the study of existence, uniqueness, differentiability of solutions of ordinary and partial differential equations and the integral equations and inequalities, etc, see [9] and the references therein. In the study of the stability of Ulam-Hyers type of functional equations, the theory of Picard operators plays a very powerful tool.

\footnotetext{
${ }^{*}$ Corresponding author.

E-mail addresses: abbasmsaid@yahoo.fr (S. Abbas), benchohra@yahoo.com (M. Benchohra), hamidi.naima@yahoo.fr (N. Hamidi).

Received July 16, 2018; Accepted December 4, 2018.
}

(C) 2018 Journal of Nonlinear Functional Analysis 
What we need is to transform the functional equation under consideration to a fixed point equation. Then, we immediately get the stability of Ulam-Hyers type of the desired equation because the Picard operator is $c$-weakly. Note that, it is not always possible to transform a functional equation or a differential equation into a fixed point problem. Indeed, this point shows the weakness of this theory. In [10], Rus used the uniform Picard operators to study the stability problems of Ulam-Hyers type. Hernández and O'Regan in [11] initially studied a class of new abstract semilinear differential equations with impulsive in which there is no instantaneous impulses in a PC-normed Banach space. Meanwhile, many authors studied other new classes of equations in which there is no instantaneous impulses; see [12, 13, 14, 15] and the references therein.

Motivated by these works, we investigate the existence and the Ulam stabilities by using Picard operators theory of the following abstract fractional differential inclusions with not instantaneous impulses

$$
\left\{\begin{array}{l}
{ }^{c} D_{s_{k}}^{r} u(t) \in B u(t)+F(t, u(t)) ; \text { if } t \in I_{k}, k=0, \ldots, m, \\
u(t)=g_{k}\left(t, u\left(t_{k}^{-}\right)\right) ; \text {if } t \in J_{k}, k=1, \ldots, m, \\
u(0)=u_{0} \in E
\end{array}\right.
$$

where $I_{0}:=\left[0, t_{1}\right], J_{k}:=\left(t_{k}, s_{k}\right], I_{k}:=\left(s_{k}, t_{k+1}\right] ; k=1, \ldots, m,{ }^{c} D_{s_{k}}^{r}$ is the Caputo fractional derivative of order $r \in(0,1], 0=s_{0}<t_{1} \leq s_{1} \leq t_{2}<\cdots<s_{m-1} \leq t_{m} \leq s_{m} \leq t_{m+1}=a, g_{k}: J_{k} \times E \rightarrow E ; k=1, \ldots, m$ are given continuous functions, $F: I_{k} \times E \rightarrow \mathscr{P}(E) ; k=0, \ldots, m$ is a closed valued multivalued map. Here, we denote by $\mathscr{P}(E)$ the family of all subsets of a (real or complex) separable Banach space $E$ and by $B$ the infinitesimal generator of a compact analytic semigroup of uniformly bounded linear operators $\{T(t) ; t>0\}$ in $E$.

We present sufficient conditions to guarantee the existence of mild solutions and the Ulam stabilities for the abstract impulsive problem (1.1). Also, we provide an example to illustrate our abstract results. To our knowledge, the Ulam stablity of semilinear fractional differential inclusions with noninstantanous impulses by means of the Picard operators have not been considered yet in the literature. So, the present paper initiates the application of Picard operators for the Ulam stability of such class of new problems.

\section{PRELIMINARIES}

We introduce, in this section, definitions, notations and preliminary facts which will be needed throughout this paper. Let $J=[0, a] ; a>0$. We denote by $L^{1}(J)$ the space of Bochner-integrable functions $u: J \rightarrow E$ with the following norm

$$
\|u\|_{L^{1}}=\int_{0}^{a}\|u(t)\|_{E} d t
$$

where $\|\cdot\|_{E}$ denotes a suitable complete norm on the separable Banach space $E$. Also, as usual, we denote by $A C(J)$ and $\mathscr{C}:=C(J)$ the space of absolutely continuous functions from $J$ into $E$ and the Banach space of all continuous functions from $J$ into $E$ with the norm

$$
\|u\|_{\infty}=\sup _{t \in J}\|u(t)\|_{E}
$$

respectively. Now, we consider the Banach space $P C$ given by

$$
P C=\left\{u: J \rightarrow E: u \in \mathscr{C}\left(I_{0} \cup \cup_{k=1}^{m}\left(t_{k}, t_{k+1}\right)\right), u\left(t_{k}^{-}\right)=u\left(t_{k}\right)\right\},
$$


with the norm

$$
\|u\|_{P C}=\sup _{t \in J}\|u(t)\|_{E}
$$

Let $r>0$. The left-sided mixed Riemann-Liouville integral of order $r$ of the function $u \in L^{1}(J)$ has the form

$$
\left(I_{0}^{r} u\right)(t)=\frac{1}{\Gamma(r)} \int_{0}^{t}(t-\tau)^{r-1} u(\tau) d \tau,
$$

where $\Gamma(\cdot)$ denotes the Euler's Gamma function given by $\Gamma(\varsigma)=\int_{0}^{\infty} t^{\varsigma^{-1}} e^{-t} d t ; \varsigma>0$. In particular,

$$
\left(I_{0}^{0} u\right)(t)=u(t),\left(I_{0}^{1} u\right)(t)=\int_{0}^{t} u(\tau) d \tau ; \text { for almost all } t \in J
$$

For instance, $I_{0}^{r} u$ exists for all $r \in(0, \infty)$, when $u \in L^{1}(J)$ and $\left(I_{0}^{r} u\right) \in C(J)$ when $u \in C(J)$.

Example 2.1. Let $\lambda \in(-1,0) \cup(0, \infty), r \in(0, \infty)$ and $h(t)=t^{\lambda} ; t \in J$. We have $h \in L^{1}(J)$, and

$$
\left(I_{0}^{r} h\right)(t)=\frac{\Gamma(1+\lambda)}{\Gamma(1+\lambda+r)} t^{\lambda+r} ; \text { for almost all } t \in J .
$$

Definition 2.2. [16] Let $r \in(0,1]$ and $u \in L^{1}(J)$. The Caputo fractional-order derivative of order $r$ of the function $u$ is defined by

$$
{ }^{c} D_{0}^{r} u(t)=\left(I_{\theta}^{1-r} \frac{d}{d t} u\right)(t)=\frac{1}{\Gamma(1-r)} \int_{0}^{t}(t-\tau)^{-r} \frac{d}{d \tau} u(\tau) d \tau
$$

Example 2.3. Let $\lambda \in(-1,0) \cup(0, \infty)$ and $r \in(0,1]$. Then

$$
{ }^{c} D_{0}^{r} t^{\lambda}=\frac{\Gamma(1+\lambda)}{\Gamma(1+\lambda-r)} t^{\lambda-r} ; \text { for almost all } t \in J .
$$

Let $a_{1} \in[0, a], J_{1}=\left(a_{1}, a\right], r>0$. For $u \in L^{1}\left(J_{1}\right)$, the expression

$$
\left(I_{a^{+}}^{r} u\right)(t)=\frac{1}{\Gamma(r)} \int_{a_{1}^{+}}^{t}(t-\tau)^{r-1} u(\tau) d \tau
$$

is called the left-sided mixed Riemann-Liouville integral of the function $u$ of arbitrary orders $r$.

Definition 2.4. [16] For $u \in L^{1}\left(J_{1}\right)$, where $\frac{d}{d t} u$ is Bochner integrable on $J_{1}$, the Caputo fractional order derivative of order $r$ of the function $u$ is defined by

$$
\left({ }^{c} D_{a^{+}}^{r} u\right)(t)=\left(I_{a^{+}}^{1-r} \frac{d}{d t} u\right)(t)
$$

In the following, we denote by $(X, d)$ a metric space and by $\mathscr{P}_{c l}(X)$ and $\mathscr{P}_{c p}(X)$ the following sets

$$
\mathscr{P}_{c l}(X)=\{Y \in \mathscr{P}(X): Y \text { is closed }\} \text { and } \mathscr{P}_{c p}(X)=\{Y \in \mathscr{P}(X): Y \text { is compact }\} \text {. }
$$

A multivalued map $G: E \rightarrow \mathscr{P}(E)$ has convex (closed) values if $G(x)$ is convex (closed) for all $x \in E$. We say that $G$ is bounded on bounded sets if $G(B)$ is bounded in $E$ for each bounded set $B$ of $E$, i.e.,

$$
\sup _{x \in B}\left\{\sup \left\{\|u\|_{E}: u \in G(x)\right\}\right\}<\infty .
$$

Finally, $G$ has a fixed point if there exists $x \in E$ with $x \in G(x)$.

For each $u \in E$, let the set $S_{F \circ u}$ known as the set of selectors from $F$ defined by

$$
S_{F \circ u}=\left\{v \in L^{1}(J): v(t) \in F(t, u(t)) \text {, a.e. } t \in J\right\} .
$$


We refer to the books of Deimling [17], Górniewicz [18] and Hu and Papageorgiou [19] for more information about multivalued maps.

Denote by $H_{d}, H_{d}: \mathscr{P}(X) \times \mathscr{P}(X) \longrightarrow \mathbb{R}_{+} \cup\{\infty\}$. The distance between the two sets $\mathscr{A}$ and $\mathscr{B}$ is defined by

$$
H_{d}(\mathscr{A}, \mathscr{B})=\max \left\{\sup _{a \in \mathscr{A}} d(a, \mathscr{B}), \sup _{b \in \mathscr{B}} d(\mathscr{A}, b)\right\}
$$

where $d(\mathscr{A}, b)=\inf _{a \in \mathscr{A}} d(a, b)$ and $d(a, \mathscr{B})=\inf _{b \in \mathscr{B}} d(a, b)$. Then the ordered pair $\left(\mathscr{P}_{b, c l}(X), H_{d}\right)$ is a metric space and $\left(\mathscr{P}_{c l}(X), H_{d}\right)$ is a generalized (complete) metric space, see [20].

Definition 2.5. [19] Let $G: J \rightarrow \mathscr{P}_{c l}(E)$ be a multivalued map. $G$ is said to be measurable if the function $Y: J \rightarrow E$ defined by

$$
Y(t)=d(x, G(t))=\inf \left\{\|x-y\|_{E}: y \in G(t)\right\}
$$

is measurable for each $x \in E$, where $d$ is the metric induced by $E$.

Lemma 2.6. (Bochner's Theorem) [21] A measurable function $u: J \rightarrow E$ is Bochner integrable if $\|u\|_{E}$ is Lebesgue integrable.

In what follows, we will state some basic definitions and results about Picard operators [9, 22]. Let $A: X \rightarrow X$ be an operator, where $(X, d)$ is a metric space. $F_{A}$ denotes the set of all fixed points of $A$. Also, the iterate operators of $A$ is denoted by $A^{0}:=1_{X}, A^{1}:=A, \ldots, A^{n+1}:=A^{n} \circ A$, where $n \in \mathbb{N}$.

Definition 2.7. $[9,22]$ An operator $A: X \rightarrow X$ is said to be a Picard operator $(P O)$ if there exists $x^{*} \in X$ satisfying

(i) $F_{A}=\left\{x^{*}\right\}$;

(ii) The sequence $\left(A^{n}\left(x_{0}\right)\right)_{n \in \mathbb{N}}$ converges to $x^{*}$ for all $x_{0} \in X$.

Definition 2.8. $[9,22]$ The operator $A: X \rightarrow X$ is said to be a weakly Picard operator (WPO) if the sequence $\left(A^{n}(x)\right)_{n \in \mathbb{N}}$ converges for all $x \in X$ and its limit, which may depend on $x$, is a fixed point of $A$.

Definition 2.9. $[9,22]$ Let $A$ be a weakly Picard operator. We define the operator $A^{\infty}: X \rightarrow X$ by

$$
A^{\infty}(x)=\lim _{n \rightarrow \infty} A^{n}(x) .
$$

Remark 2.10. It is easy to see that $F_{A}=A^{\infty}(X)$.

Definition 2.11. $[9,22]$ The weakly Picard operator $A$ is called a c-weakly Picard operator if

$$
d\left(x, A^{\infty}(x)\right) \leq c d(x, A(x)), x \in X, c>0 .
$$

In the multivalued case, we have the following concept.

Definition 2.12. $[23,24]$ Let $(X, d)$ be a metric space and let $F: X \rightarrow \mathscr{P}_{c l}(X)$ be a multivalued operator. $F$ is said to be a multivalued weakly Picard operator (MWPO), if for each $u \in X$ and each $v \in F(x)$, there exists a sequence $\left(u_{n}\right)_{n \in \mathbb{N}}$ satisfying

(i) $u_{0}=u, u_{1}=v$,

(ii) $u_{n+1} \in F\left(u_{n}\right)$, for each $n \in \mathbb{N}$,

(iii) $\left(u_{n}\right)_{n \in \mathbb{N}}$ converges to a fixed point of $F$. 
Remark 2.13. A sequence $\left(u_{n}\right)_{n \in \mathbb{N}}$ satisfying condition $(i)$ and $(i i)$ in the above Definition is called a sequence of successive approximations of $F$ starting from $(x, y) \in \operatorname{Graph}(F)$.

If $F: X \rightarrow \mathscr{P}_{c l}(X)$ is a (MWPO), then we define $F_{1}: \operatorname{Graph}(F) \rightarrow \mathscr{P}(F i x(F))$ by the formula $F_{1}(x, y):=\{u \in F i x(F)$. There exists a sequence of successive approximations of $F$ starting from $(x, y)$ that converges to $u\}$.

Definition 2.14. $[23,24]$ Let $(X, d)$ be a metric space and let $\Psi:[0, \infty) \rightarrow[0, \infty)$ be an increasing function, which is continuous at 0 and $\Psi(0)=0$. Then $F: X \rightarrow \mathscr{P}_{c l}(X)$ is said to be a multivalued $\Psi$-weakly Picard operator ( $\Psi-\mathrm{MWPO})$ if it is a multivalued weakly Picard operator and there exists a selection $A^{\infty}: \operatorname{Graph}(F) \rightarrow \operatorname{Fix}(F)$ of $F^{\infty}$ such that

$$
d\left(u, A^{\infty}(u, v)\right) \leq \Psi(d(u, v)) ; \text { for all }(u, v) \in \operatorname{Graph}(F) .
$$

If there exists $c>0$ such that $\Psi(t)=c t$, for each $t \in[0, \infty)$, then $F$ is called a multivalued c-weakly Picard operator $(c-M W P O)$.

Definition 2.15. [25] A multivalued operator $N: X \rightarrow \mathscr{P}_{c l}(X)$ is called

a) $\gamma$-Lipschitz if and only if there exists $\gamma \geq 0$ such that

$$
H_{d}(N(u), N(v)) \leq \gamma d(u, v) \text { for each } u, v \in X,
$$

b) a multivalued $\gamma$-contraction if and only if it is $\gamma$-Lipschitz with $\gamma \in[0,1)$.

Following [26, 27], we introduce now the definition of mild solution to (1.1).

Definition 2.16. A function $u: J \rightarrow E$ is said to be a mild solution of (1.1) if there exists $f \in S_{F \circ u}$, such that $u$ satisfies

$$
\left\{\begin{array}{l}
u(t)=S_{r}(t) u_{0}+\int_{0}^{t}(t-\tau)^{r-1} T_{r}(t-\tau) f(\tau) d \tau ; \text { if } t \in\left[0, t_{1}\right] \\
u(t)=S_{r}\left(t-s_{k}\right) g_{k}\left(s_{k}, u\left(t_{k}^{-}\right)\right) \\
+\int_{s_{k}}^{t}(t-\tau)^{r-1} T_{r}(t-\tau) f(\tau) d \tau ; \text { if } t \in I_{k}, k=1, \ldots, m, \\
u(t)=g_{k}\left(t, u\left(t_{k}^{-}\right)\right) ; \text {if } t \in J_{k}, k=1, \ldots, m, \\
u(0)=u_{0},
\end{array}\right.
$$

where

$$
\begin{gathered}
S_{r}(t)=\int_{0}^{\infty} \xi_{r}(\theta) T\left(t^{r} \theta\right) d \theta, \\
T_{r}(t)=r \int_{0}^{\infty} \theta \xi_{r}(\theta) T\left(t^{r} \theta\right) d \theta, \\
\xi_{r}(\theta)=\frac{1}{r} \theta^{-1-\frac{1}{r}} \bar{w}_{r}\left(\theta^{-\frac{1}{r}}\right) \geq 0,
\end{gathered}
$$

and

$$
\bar{w}_{r}(\theta)=\frac{1}{\pi} \sum_{n=0}^{\infty}(-1)^{n-1} \theta^{-n r-1} \frac{\Gamma(n r+1)}{n !} \sin (n r \pi) ; \theta \in(0, \infty) .
$$

$\xi_{q}$ is a probability density function on $(0, \infty)$, that is, $\int_{0}^{\infty} \xi_{r}(\theta) d \theta=1$. 
Remark 2.17. It is not difficult to verify that, for $v \in[0,1]$,

$$
\int_{0}^{\infty} \theta^{v} \xi_{r}(\theta) d \theta=\int_{0}^{\infty} \theta^{-r v} \bar{w}_{r}(\theta) d \theta=\frac{\Gamma(1+v)}{\Gamma(1+r v)} .
$$

Lemma 2.18. [27] For any $t \geq 0$, operators $S_{r}(t)$ and $T_{r}(t)$ have the following properties:

(a) For any fixed $t \geq 0, S_{r}$ and $T_{r}$ are linear and bounded operators, i.e., for any $u \in E$,

$$
\left\|S_{r}(t) u\right\|_{E} \leq M\|u\|_{E},\left\|T_{r}(t) u\right\|_{E} \leq \frac{M}{\Gamma(r)}\|u\|_{E} .
$$

(b) $\left\{S_{r}(t) ; t \geq 0\right\}$ and $\left\{T_{r}(t) ; t \geq 0\right\}$ are strongly continuous.

Remark 2.19. By using Definition 2.16, solutions of problem (1.1) are solutions of the fixed point inclusion $u \in N(u)$, where $N: P C \rightarrow \mathscr{P}(P C)$ is the multivalued operator defined by

$$
(N u)(t)=\left\{h \in P C:\left\{\begin{array}{l}
h(t)=S_{r}(t) u_{0} \\
+\int_{0}^{t}(t-\tau)^{r-1} T_{r}(t-\tau) f(\tau) d \tau ; \\
\text { if } t \in\left[0, t_{1}\right] \\
h(t)=S_{r}\left(t-s_{k}\right) g_{k}\left(s_{k}, u\left(t_{k}^{-}\right)\right) \\
+\int_{s_{k}}^{t}(t-\tau)^{r-1} T_{r}(t-\tau) f(\tau) d \tau ; \\
\text { if } t \in I_{k}, k=1, \ldots, m \\
h(t)=g_{k}\left(t, u\left(t_{k}^{-}\right)\right) ; \text {if } t \in J_{k}, k=1, \ldots, m
\end{array} ; f \in S_{F \circ u}\right\}\right.
$$

Now, we consider the Ulam stability for fixed point inclusion $u \in N(u)$. Let $\varepsilon>0, \Psi \geq 0$ and $\Phi: J \rightarrow$ $[0, \infty)$ be a continuous function. We consider the following inequalities

$$
\begin{aligned}
& \left\{\begin{array}{l}
d(u, N(u)) \leq \varepsilon ; \text { if } t \in I_{k}, k=0, \ldots, m, \\
\left\|u(t)-g_{k}\left(t, u\left(t_{k}^{-}\right)\right)\right\|_{E} \leq \varepsilon ; \text { if } t \in J_{k}, k=1, \ldots, m,
\end{array}\right. \\
& \left\{\begin{array}{l}
d(u, N(u)) \leq \Phi(t) ; \text { if } t \in I_{k}, k=0, \ldots, m, \\
\left\|u(t)-g_{k}\left(t, u\left(t_{k}^{-}\right)\right)\right\|_{E} \leq \Psi ; \text { if } t \in J_{k}, k=1, \ldots, m,
\end{array}\right. \\
& \left\{\begin{array}{l}
d(u, N(u)) \leq \varepsilon \Phi(t) ; \text { if } t \in I_{k}, k=0, \ldots, m, \\
\left\|u(t)-g_{k}\left(t, u\left(t_{k}^{-}\right)\right)\right\|_{E} \leq \varepsilon \Psi ; \text { if } t \in J_{k}, k=1, \ldots, m
\end{array}\right.
\end{aligned}
$$

Definition 2.20. $[3,13,15]$ The fixed point inclusion $u \in N(u)$ is Ulam-Hyers stable if there exists a real number $c_{N, g_{k}}>0$ such that for each $\varepsilon>0$ and for each solution $u \in P C$ of (2.1) there exists $v \in P C$ such that $v \in N(v)$ with

$$
\|u(t)-v(t)\|_{P C} \leq \varepsilon c_{N, g_{k}} ; t \in J .
$$

Definition 2.21. $[3,13,15]$ The fixed point inclusion $u \in N(u)$ is generalized Ulam-Hyers stable if there exists $c_{N, g_{k}}: C([0, \infty),[0, \infty))$ with $c_{N, g_{k}}(0)=0$ such that for each $\varepsilon>0$ and for each solution $u \in P C$ of (2.1) there exists $v \in P C$ such that $v \in N(v)$ with

$$
\|u(t)-v(t)\|_{P C} \leq c_{N, g_{k}}(\varepsilon) ; t \in J .
$$


Definition 2.22. [3, 13, 15] The fixed point inclusion is Ulam-Hyers-Rassias stable with respect to $(\Phi, \Psi)$ if there exists a real number $c_{N, g_{k}, \Phi}>0$ such that for each $\varepsilon>0$ and for each solution $u \in P C$ of (2.3) there exists $v \in P C$ such that $v \in N(v)$ with

$$
\|u(t)-v(t)\|_{P C} \leq \varepsilon c_{N, g_{k}, \Phi}(\Psi+\Phi(t)) ; t \in J .
$$

Definition 2.23. [3, 13, 15] Problem (1.1) is generalized Ulam-Hyers-Rassias stable with respect to $(\Phi, \Psi)$ if there exists a real number $c_{N, g_{k}, \Phi}>0$ such that for each solution $u \in P C$ of (2.2) there exists $v \in P C$ such that $v \in N(v)$ with $\|u(t)-v(t)\|_{P C} \leq c_{N, g_{k}, \Phi}(\Psi+\Phi(t)) ; t \in J$.

Remark 2.24. It is clear that: (i) Definition $2.20 \Rightarrow$ Definition 2.21, (ii) Definition $2.22 \Rightarrow$ Definition 2.23, (iii) Definition 2.22 for $\Phi()=.\Psi=1 \Rightarrow$ Definition 2.20.

Lemma 2.25. (Covitz-Nadler) [25] Let $(X, d)$ be a complete metric space. If $A: X \rightarrow \mathscr{P}_{c l}(X)$ is a contraction, then A has fixed points.

Now we present two important characterizations from the viewpoint of the Ulam-Hyers stability.

Lemma 2.26. [28] Let $(X, d)$ be a metric space. If $A: X \rightarrow \mathscr{P}_{c l}(X)$ is a $(\Psi-M W P O)$, then the fixed point inclusion $u \in A(u)$ is generalized Ulam-Hyers stable. In particular, if $A$ is $(c-M W P O)$, then $u \in A(u)$ is Ulam-Hyers stable.

Lemma 2.27. $[23,29]$ Let $(X, d)$ be a Banach space. If an operator $A: X \rightarrow \mathscr{P}_{c l}(X)$ is a q-contraction, then $A$ is a $(c-M W P O)$ with $c=\frac{1}{1-q}$.

From Lemmas 2.25, 2.26 and 2.27 we have the following lemma.

Lemma 2.28. $[23,24]$ Let $(X, d)$ be a Banach space. If an operator $A: X \rightarrow \mathscr{P}_{c l}(X)$ is a q-contraction. Then the following hold:

(i) The operator A has fixed points;

(ii) The operator $A$ is a $(c-M W P O)$ with $c=\frac{1}{1-q}$; and

(iii) The fixed point inclusion $u \in A(u)$ is Ulam-Hyers stable.

In the sequel, we will make use of the following Theorem [[30],Th. 8.6.3] of Aubin and Frankowska for the representation of extremal points of closure of set-valued integral.

Theorem 2.29. [30] Let $\mathfrak{P}=(\Omega, F, \mathbb{P})$ be a nonatomic probability space, and let $H$ be a measurable set-valued map from $\Omega$ to subsets of $\mathbb{R}^{n}$ with nonempty closed images. Then the following hold:

(a) The set-valued integral $\int_{\Omega} H d \mathbb{P}$ is convex.

(b) If $H$ is integrably bounded, then the integral $\int_{\Omega} H d \mathbb{P}$ is also compact.

By the above theorem, we conclude with a representation of points in a set-valued integral in the following lemma.

Lemma 2.30. [30] Let $F: J \times E \rightarrow \mathscr{P}_{c l}(E)$ be such that $x \longmapsto F(x, u)$ is measurable for each $u \in E$. Then the following hold:

(a) The set-valued integral $I_{\theta}^{r} F$ is convex.

(b) If $F$ is integrably bounded, then the integral $I_{\theta}^{r} F$ is compact. 
Lemma 2.31. [31] Let $\omega$ be a nonnegative and locally integrable function on $J$ and $v: J \rightarrow[0, \infty)$ be a real function. If there are constants $0<r<1$ and $c>0$ satisfying

$$
v(t) \leq \omega(t)+c \int_{0}^{t} v(\tau)(t-\tau)^{-r} d \tau
$$

then there exists a constant $\delta=\delta(r)$ satisfying

$$
v(t) \leq \omega(t)+c \delta \int_{0}^{t} \omega(\tau)(t-\tau)^{-r} d \tau
$$

for every $t \in J$.

\section{RESUlTS ON EXISTENCE AND ULAM STABILITIES}

In this section, we present the existence of mild solutions and we discuss the conditions under which problem (1.1) is stable in the sense of Ulam. Before stating and proving our main results, let us introduce the following hypotheses.

$\left(H_{1}\right)$ The multifunction $F: I_{k} \times E \longrightarrow \mathscr{P}(E)$ satisfies

$$
F(\cdot, u): I_{k} \rightarrow \mathscr{P}_{c p}(E) \text { is measurable for each } u \in E, k=0, \ldots, m,
$$

and

$$
F(t, u) \subset B(0,1):=\left\{u \in E:\|u\|_{E}<1\right\} ;
$$

for each $u \in E$ and all $t \in I_{k} ; k=0, \ldots, m$.

$\left(H_{2}\right)$ There exists a constant $l_{F}>0$ such that

$$
H_{d}(F(t, u), F(t, v)) \leq l_{F}\|u-v\|_{E}
$$

for each $u, v \in E$ and each $t \in I_{k} ; k=0, \ldots, m$.

$\left(H_{3}\right)$ There exist constants $0<l_{g_{k}}<1 ; k=1, \ldots, m$ such that

$$
\left\|g_{k}\left(t, u\left(t_{k}^{-}\right)\right)-g_{k}\left(t, \bar{u}\left(t_{k}^{-}\right)\right)\right\|_{E} \leq l_{g_{k}}\|u(t)-\bar{u}(t)\|_{E},
$$

for each $t \in J_{k}$, and each $u, \bar{u} \in P C, k=1, \ldots, m$.

$\left(H_{4}\right)$ There exists $\lambda_{\Phi}>0$ such that, for each $t \in J$,

$$
I_{s_{k}}^{r} \Phi(t) \leq \lambda_{\Phi} \Phi(t) ; k=0, \ldots, m .
$$

Remark 3.1. From $\left(H_{1}\right)$, we see that the set $S_{F \circ u}$ is nonempty for each $u \in P C$ because $F$ has a measurable selection (see [32], Theorem III.6).

Theorem 3.2. Assume that $\left(H_{1}\right)-\left(H_{3}\right)$ are satisfied. If

$$
\ell_{F}:=M l_{g}+\frac{M l_{F} a^{r}}{\Gamma(r)}<1,
$$

where $l_{g}=\max _{k=1, \ldots, m} l_{g_{k}}$, then problem (1.1) has mild solutions on $J$, and $N$ is a $\left(k_{N}-M W P O\right)$ with $k_{N}=$ $\frac{1}{1-\ell_{F}}$. Moreover the fixed point inclusion $u \in N(u)$ is Ulam-Hyers stable. Furthermore, if $\left(H_{4}\right)$ holds, then the fixed point inclusion $u \in N(u)$ is generalized Ulam-Hyers-Rassias stable. 
Proof. Let $N: P C \rightarrow \mathscr{P}(P C)$ be the multivalued operator defined in Remark 2.19. In view of Lemma 2.18 , for each $w \in S_{F \circ u}$ and $t \in I_{k} ; k=0, \ldots, m$, we have

$$
\begin{aligned}
\int_{s_{k}}^{t}\left\|(t-\tau)^{r-1} T_{r}(t-\tau) w(\tau)\right\|_{E} d \tau & \leq \frac{M}{\Gamma(r)} \int_{s_{k}}^{t}(t-\tau)^{r-1}\|w(\tau)\|_{E} d \tau \\
& =M I_{\theta_{k}}^{r}\|w(t)\|_{E} .
\end{aligned}
$$

Thus, $\left\|T_{r}(t-\tau) w(\tau)\right\|_{E}$ is Lebesgue integrable with respect to $\tau \in[0, t]$ for all $t \in[0, a]$. From Lemma 2.6, it follows that $T_{r}(t-\tau) w(\tau)$ is Bochner integrable with respect to $\tau \in[0, t]$ for all $t \in[0, a]$. In addition, in view of the fact that the functions $g_{k} ; k=1, \ldots, m$, are continuous and the operators $\left\{S_{r}(t) ; t \geq 0\right\}$ and $\left\{T_{r}(t) ; t \geq 0\right\}$ are strongly continuous, and Theorem 2 in Rybiński [33], we have that the function $h \in P C$ defined by

$$
\left\{\begin{array}{l}
h(t)=S_{r}(t) u_{0}+\int_{0}^{t}(t-\tau)^{r-1} T_{r}(t-\tau) w(\tau) d \tau ; \text { if } t \in\left[0, t_{1}\right], \\
h(t)=S_{r}\left(t-s_{k}\right) g_{k}\left(s_{k}, u\left(t_{k}^{-}\right)\right) \\
+\int_{s_{k}}^{t}(t-\tau)^{r-1} T_{r}(t-\tau) w(\tau) d \tau ; \text { if } t \in I_{k}, k=1, \ldots, m, \\
h(t)=g_{k}\left(t, u\left(t_{k}^{-}\right)\right) ; \text {if } t \in J_{k}, k=1, \ldots, m,
\end{array}\right.
$$

has the property $h \in N(u)$. We shall show that $N$ satisfies the assumptions of Lemma 2.28. From $\left(H_{1}\right)$, via Lemma 2.30, we have that $N(u)$ is a compact set, for each $u \in P C$. It remains to show that $N$ is a contractive multivalued operator. Let $u \in P C$ and $\left(h_{n}\right)_{n \geq 0} \in N(u)$ such that $h_{n} \longrightarrow h$ in $P C$. Then, $h \in P C$ and there exists $f_{n} \in S_{F, u}$ such that, for each $t \in J$,

$$
\left\{\begin{array}{l}
h_{n}(t)=S_{r}(t) u_{0}+\int_{0}^{t}(t-\tau)^{r-1} T_{r}(t-\tau) f_{n}(\tau) d \tau ; \text { if } t \in\left[0, t_{1}\right] \\
h_{n}(t)=S_{r}\left(t-s_{k}\right) g_{k}\left(s_{k}, u\left(t_{k}^{-}\right)\right) \\
+\int_{s_{k}}^{t}(t-\tau)^{r-1} T_{r}(t-\tau) f_{n}(\tau) d \tau ; \text { if } t \in I_{k}, k=1, \ldots, m \\
h_{n}(t)=g_{k}\left(t, u\left(t_{k}^{-}\right)\right) ; \text {if } t \in J_{k}, k=1, \ldots, m .
\end{array}\right.
$$

Using the fact that $F$ has compact values and from $\left(H_{1}\right)$, we may pass to a subsequence if necessary to get that $f_{n}($.$) converges to f$ in $L^{1}\left(I_{k}\right) ; k=0, \ldots, m$, and hence $f \in S_{F \circ u}$. Then, for each $t \in I_{k} ; k=0, \ldots, m$, we have $h_{n}(t) \longrightarrow h(t)$, where

$$
\left\{\begin{array}{l}
h(t)=S_{r}(t) u_{0}+\int_{0}^{t}(t-\tau)^{r-1} T_{r}(t-\tau) f(\tau) d \tau ; \text { if } t \in\left[0, t_{1}\right], \\
h(t)=S_{r}\left(t-s_{k}\right) g_{k}\left(s_{k}, u\left(t_{k}^{-}\right)\right) \\
+\int_{s_{k}}^{t}(t-\tau)^{r-1} T_{r}(t-\tau) f(\tau) d \tau ; \text { if } t \in I_{k}, k=1, \ldots, m, \\
h(t)=g_{k}\left(t, u\left(t_{k}^{-}\right)\right) ; \text {if } t \in J_{k}, k=1, \ldots, m .
\end{array}\right.
$$

So, $h \in N(u)$. 
Next, we show that $N$ is a contraction multivalued operator. Let $u, \bar{u} \in P C$ and $h \in N(u)$. Then, there exists $f \in S_{F \circ u}$ such that, for each $t \in J$,

$$
\left\{\begin{array}{l}
h(t)=S_{r}(t) u_{0}+\int_{0}^{t}(t-\tau)^{r-1} T_{r}(t-\tau) f(\tau) d \tau ; \text { if } t \in\left[0, t_{1}\right] \\
h(t)=S_{r}\left(t-s_{k}\right) g_{k}\left(s_{k}, u\left(t_{k}^{-}\right)\right) \\
+\int_{s_{k}}^{t}(t-\tau)^{r-1} T_{r}(t-\tau) f(\tau) d \tau ; \text { if } t \in I_{k}, k=1, \ldots, m, \\
h(t)=g_{k}\left(t, u\left(t_{k}^{-}\right)\right) ; \text {if } t \in J_{k}, k=1, \ldots, m .
\end{array}\right.
$$

From $\left(H_{2}\right)$, it follows that

$$
H_{d}(F(t, u(t)), F(t, \bar{u}(t))) \leq l_{F}\|u(t)-\bar{u}(t)\|_{E} .
$$

Hence, there exists $w(t) \in F(t, \bar{u}(t))$ such that

$$
\|f(t)-w(t)\|_{E} \leq l_{F}\|u(t)-\bar{u}(t)\|_{E} ; t \in I_{k} ; k=0, \ldots, m .
$$

We consider $U: I_{k} \rightarrow \mathscr{P}(E)$ given by

$$
U(t)=\left\{w \in P C:\|f(t)-w(t)\|_{E} \leq l_{F}\|u(t)-\bar{u}(t)\|_{E}\right\} .
$$

Since the multivalued operator $U(t) \cap F(t, \bar{u}(t))$ is measurable (see Proposition III.4 in [32]), there exists a function $\bar{f}(t)$, which is a measurable selection for $u$. So, $\bar{f}(t) \in F(t, \bar{u}(t))$, and for each $t \in I_{k} ; k=$ $0, \ldots, m$, we have

$$
\|f(t)-\bar{f}(t)\|_{E} \leq l_{F}\|u(t)-\bar{u}(t)\|_{E}
$$

Let us define, for each $t \in J$,

$$
\left\{\begin{array}{l}
\bar{h}(t)=S_{r}(t) u_{0}+\int_{0}^{t}(t-\tau)^{r-1} T_{r}(t-\tau) \bar{f}(\tau) d \tau ; \text { if } t \in\left[0, t_{1}\right] \\
\bar{h}(t)=S_{r}\left(t-s_{k}\right) g_{k}\left(s_{k}, \bar{u}\left(t_{k}^{-}\right)\right) \\
+\int_{s_{k}}^{t}(t-\tau)^{r-1} T_{r}(t-\tau) \bar{f}(\tau) d \tau ; \text { if } t \in I_{k}, k=1, \ldots, m, \\
\bar{h}(t)=g_{k}\left(t, \bar{u}\left(t_{k}^{-}\right)\right) ; \text {if } t \in J_{k}, k=1, \ldots, m .
\end{array}\right.
$$

It follows that

$$
\left\{\begin{array}{l}
\|h(t)-\bar{h}(t)\|_{E} \leq \| \int_{0}^{t}(t-\tau)^{r-1} T_{r}(t-\tau) \\
\times[f(\tau)-\bar{f}(\tau)] d \tau \|_{E} ; \text { if } t \in\left[0, t_{1}\right] \\
\|h(t)-\bar{h}(t)\|_{E} \leq \| S_{r}\left(t-s_{k}\right)\left(g_{k}\left(s_{k}, u\left(s_{k}\right)\right)-g_{k}\left(s_{k}, \bar{u}\left(t_{k}^{-}\right)\right) \|_{E}\right. \\
+\left\|\int_{s_{k}}^{t}(t-\tau)^{r_{1}-1} T_{r}\left(t-s_{k}\right)[f(\tau)-\bar{f}(\tau)] d \tau\right\|_{E} ; \text { if } t \in I_{k}, k=1, \ldots, m, \\
\|h(t)-\bar{h}(t)\|_{E}=\left\|g_{k}\left(t, u\left(t_{k}^{-}\right)\right)-g_{k}\left(t, \bar{u}\left(t_{k}^{-}\right)\right)\right\|_{E} ; \text { if } t \in J_{k}, k=1, \ldots, m .
\end{array}\right.
$$


Thus,

$$
\left\{\begin{array}{l}
\|h(t)-\bar{h}(t)\|_{E} \leq \int_{0}^{t}(t-\tau)^{r-1} T_{r}(t-\tau) l_{F}\left\|T_{r}(t-\tau)(u(\tau)-\bar{u}(\tau))\right\|_{E} d \tau \\
\leq \frac{M l_{F} a^{r}}{\Gamma(r)}\|u-\bar{u}\|_{P C} ; \text { if } t \in\left[0, t_{1}\right] \\
\|h(t)-\bar{h}(t)\|_{E} \leq l_{g}\left\|S_{r}\left(t-s_{k}\right)(u(t)-\bar{u}(t))\right\|_{E} \\
+\int_{s_{k}}^{t}(t-\tau)^{r-1} l_{F}\left\|T_{r}(t-\tau)(u(\tau)-\bar{u}(\tau))\right\|_{E} d \tau \\
\leq\left(M l_{g}+\frac{M l_{F} a^{r}}{\Gamma(r)}\right)\|u-\bar{u}\|_{P C} ; \text { if } t \in I_{k}, k=1, \ldots, m \\
\|h(t)-\bar{h}(t)\|_{E} \leq l_{g}\|u-\bar{u}\|_{P C} ; \text { if } t \in J_{k}, k=1, \ldots, m
\end{array}\right.
$$

This implies that $\|h-\bar{h}\|_{P C} \leq \ell_{F}\|u-\bar{u}\|_{P C}$. By an analogous relation, obtained by interchanging the roles of $u$ and $\bar{u}$, it follows that

$$
H_{d}(N(u), N(\bar{u})) \leq \ell_{F}\|u-\bar{u}\|_{P C} .
$$

Using (3.1), we conclude that $N$ is a $\ell_{F}$-contraction. Consequently, by Lemma 2.28, $N$ has a fixed point which is mild solution on $J$ of (1.1), $N$ is a $\left(c_{N}-M W P O\right)$ with $c_{N}=\frac{1}{1-\ell_{F}}$ and the fixed point inclusion $u \in N(u)$ is Ulam-Hyers stable.

Now, we prove the generalized Ulam-Hyers-Rassias stability of $u \in N(u)$. Let $v$ be a solution of (1.1). Then, there exists $f_{v} \in S_{F, v}$ such that

$$
\left\{\begin{array}{l}
v(t)=S_{r}(t) u_{0}+\int_{0}^{t}(t-\tau)^{r-1} T_{r}(t-\tau) f_{v}(\tau) d \tau ; \text { if } t \in\left[0, t_{1}\right], \\
v(t)=S_{r}\left(t-s_{k}\right) g_{k}\left(s_{k}, v\left(t_{k}^{-}\right)\right) \\
+\int_{s_{k}}^{t}(t-\tau)^{r-1} T_{r}(t-\tau) f_{v}(\tau) d \tau ; \text { if } t \in I_{k}, k=1, \ldots, m, \\
v(t)=g_{k}\left(t, v\left(t_{k}^{-}\right)\right) ; \text {if } t \in J_{k}, k=1, \ldots, m .
\end{array}\right.
$$

Let $u \in P C$ be a solution of (2.2). For each $t \in I_{k}, k=0, \ldots, m$, we have

$$
\begin{aligned}
\|u(t)-v(t)\|_{E} & \leq d(u, N(u))+d(v, N(u)) \\
& \leq \Phi(t)+d(v, N(u)),
\end{aligned}
$$

and for each $t \in J_{k}, k=1, \ldots, m$, we have

$$
\begin{aligned}
\|u(t)-v(t)\|_{E} & \leq\left\|u(t)-g_{k}\left(t, u\left(t_{k}^{-}\right)\right)\right\|_{E}+\left\|g_{k}\left(t, u\left(t_{k}^{-}\right)\right)-g_{k}\left(t, v\left(t_{k}^{-}\right)\right)\right\|_{E} \\
& \leq \Psi+\left\|g_{k}\left(t, u\left(t_{k}^{-}\right)\right)-g_{k}\left(t, v\left(t_{k}^{-}\right)\right)\right\|_{E} .
\end{aligned}
$$

Letting $h \in N(u)$, we see that there exists $f \in S_{F, u}$ such that

$$
\left\{\begin{array}{l}
h(t)=S_{r}(t) u_{0}+\int_{0}^{t}(t-\tau)^{r-1} T_{r}(t-\tau) f(\tau) d \tau ; \text { if } t \in\left[0, t_{1}\right], \\
h(t)=S_{r}\left(t-s_{k}\right) g_{k}\left(s_{k}, u\left(t_{k}^{-}\right)\right) \\
+\int_{s_{k}}^{t}(t-\tau)^{r-1} T_{r}(t-\tau) f(\tau) d \tau ; \text { if } t \in I_{k}, k=1, \ldots, m, \\
h(t)=g_{k}\left(t, u\left(t_{k}^{-}\right)\right) ; \text {if } t \in J_{k}, k=1, \ldots, m .
\end{array}\right.
$$


Then,

$$
\left\{\begin{array}{l}
\|h(t)-v(t)\|_{E} \leq \| \int_{0}^{t}(t-\tau)^{r-1} T_{r}(t-\tau) \\
\times\left[f(\tau)-f_{v}(\tau)\right] d \tau \|_{E} ; \text { if } t \in\left[0, t_{1}\right] \\
\|h(t)-v(t)\|_{E} \leq \Phi(t)+M \| g_{k}\left(s_{k}, u\left(t_{k}^{-}\right)\right)-g_{k}\left(s_{k}, v\left(t_{k}^{-}\right) \|_{E}\right. \\
+\int_{s_{k}}^{t}(t-\tau)^{r_{1}-1}\left\|T_{r}(t-\tau)\left(f(\tau)-f_{v}(\tau)\right)\right\|_{E} d \tau ; \\
\text { if } t \in I_{k}, k=1, \ldots, m .
\end{array}\right.
$$

Hence,

$$
\left\{\begin{array}{l}
\|h(t)-v(t)\|_{E} \leq \int_{0}^{t}(t-\tau)^{r-1} T_{r}(t-\tau) l_{F}\left\|T_{r}(t-\tau)(u(\tau)-v(\tau))\right\|_{E} d \tau \\
\leq \frac{M l_{F}}{\Gamma(r)} \int_{0}^{t}(t-\tau)^{r-1}\|u(\tau)-v(\tau)\|_{E} d \tau ; \text { if } t \in\left[0, t_{1}\right], \\
\|h(t)-v(t)\|_{E} \leq M l_{g}\|u(t)-v(t)\|_{E} \\
+\frac{M l_{F}}{\Gamma(r)} \int_{s_{k}}^{t}(t-\tau)^{r_{1}-1}\|u(\tau)-v(\tau)\|_{E} d \tau ; \text { if } t \in I_{k}, k=1, \ldots, m .
\end{array}\right.
$$

By an analogous relation, obtained by interchanging the roles of $u$ and $v$, it follows that

$$
\left\{\begin{array}{l}
d(v, N(u)) \leq \int_{0}^{t}(t-\tau)^{r-1} T_{r}(t-\tau) l_{F}\left\|T_{r}(t-\tau)(u(\tau)-v(\tau))\right\|_{E} d \tau \\
\leq \frac{M l_{F}}{\Gamma(r)} \int_{0}^{t}(t-\tau)^{r-1}\|u(\tau)-v(\tau)\|_{E} d \tau ; \text { if } t \in\left[0, t_{1}\right], \\
d(v, N(u)) \leq M l_{g}\|u(t)-v(t)\|_{E} \\
+\frac{M l_{F}}{\Gamma(r)} \int_{s_{k}}^{t}(t-\tau)^{r_{1}-1}\|u(\tau)-v(\tau)\|_{E} d \tau ; \text { if } t \in I_{k}, k=1, \ldots, m .
\end{array}\right.
$$

Hence, for each $t \in J$, we get

$$
\left\{\begin{array}{l}
\|u(t)-v(t)\|_{E} \leq \Phi(t)+\frac{M l_{F}}{\Gamma(r)} \int_{0}^{t}(t-\tau)^{r-1}\|u(\tau)-v(\tau)\|_{E} d \tau ; \text { if } t \in\left[0, t_{1}\right] \\
\|u(t)-v(t)\|_{E} \leq \Phi(t)+M l_{g}\|u(t)-v(t)\|_{E} \\
+\frac{M l_{F}}{\Gamma(r)} \int_{s_{k}}^{t}(t-\tau)^{r_{1}-1}\|u(\tau)-v(\tau)\|_{E} d \tau ; \text { if } t \in I_{k}, k=1, \ldots, m \\
\|u(t)-v(t)\|_{E} \leq \Psi+l_{g}\|u(t)-v(t)\|_{E} ; \text { if } t \in J_{k}, k=1, \ldots, m
\end{array}\right.
$$

For each $t \in\left[0, t_{1}\right]$, we have

$$
\|u(t)-v(t)\|_{E} \leq \Phi(t)+\frac{M l_{F}}{\Gamma(r)} \int_{0}^{t}(t-\tau)^{r-1}\|u(\tau)-v(\tau)\|_{E} d \tau .
$$

From Lemma 2.31 and $\left(H_{4}\right)$, there exists a constant $\delta_{1}:=\delta_{1}(r)$ such that

$$
\begin{aligned}
\|u(t)-v(t)\|_{E} & \leq \Phi(t)+M l_{F} \delta_{1} I_{0}^{r} \Phi(t) \\
& \leq\left(1+M l_{F} \delta_{1} \lambda_{\Phi}\right) \Phi(t) \\
& :=c_{1, N, g_{k}, \Phi} \Phi(t) .
\end{aligned}
$$

Thus, for each $t \in\left[0, t_{1}\right]$, we get

$$
\|u(t)-v(t)\|_{E} \leq c_{1, N, g_{k}, \Phi}(\Psi+\Phi(t)) .
$$


Now, for each $t \in I_{k}, k=1, \ldots, m$, we have

$$
\begin{gathered}
\|u(t)-v(t)\|_{E} \leq \Phi(t)+M l_{g}\|u(t)-v(t)\|_{E} \\
+\frac{M l_{F}}{\Gamma(r)} \int_{s_{k}}^{t}(t-\tau)^{r-1}\|u(\tau)-v(\tau)\|_{E} d \tau .
\end{gathered}
$$

It follows that

$$
\|u(t)-v(t)\|_{E} \leq \frac{1}{1-M l_{g}} \Phi(t)+\frac{M l_{F}}{\left(1-M l_{g}\right) \Gamma(r)} \int_{s_{k}}^{t}(t-\tau)^{r-1}\|u(\tau)-v(\tau)\|_{E} d \tau .
$$

Again, from Lemma 2.31 and $\left(H_{4}\right)$, there exists a constant $\delta_{2}:=\delta_{2}(r)$ such that

$$
\begin{aligned}
\|u(t)-v(t)\|_{E} & \leq \frac{1}{1-M l_{g}}\left(\Phi(t)+\frac{M l_{F} \delta_{2}}{1-M l_{g}} I_{s_{k}}^{r} \Phi(t)\right) \\
& \leq \frac{1}{1-M l_{g}}\left(1+\frac{M l_{F} \delta_{2} \lambda_{\Phi}}{1-M l_{g}}\right) \Phi(t) \\
& :=c_{2, N, g_{k}, \Phi} \Phi(t) .
\end{aligned}
$$

Hence, for each $t \in I_{k}, k=1, \ldots, m$, we get

$$
\|u(t)-v(t)\|_{E} \leq c_{2, N, g_{k}, \Phi}(\Psi+\Phi(t)) .
$$

Now, for each $t \in J_{k}, k=1, \ldots, m$, we have

$$
\|u(t)-v(t)\|_{E} \leq \Psi+l_{g}\|u(t)-v(t)\|_{E} .
$$

This gives that

$$
\|u(t)-v(t)\|_{E} \leq \frac{\Psi}{1-l_{g}}:=c_{3, N, g_{k}, \Phi} \Psi .
$$

Thus, for each $t \in J_{k}, k=1, \ldots, m$, we get

$$
\|u(t)-v(t)\|_{E} \leq c_{3, N, g_{k}, \Phi}(\Psi+\Phi(t)) .
$$

Set $c_{N, g_{k}, \Phi}:=\max _{i \in\{1,2,3\}} c_{i, N, g_{k}, \Phi}$. Hence, for each $t \in J$, we obtain

$$
\|u(t)-v(t)\|_{P C} \leq c_{N, g_{k}, \Phi}(\Psi+\Phi(t)) .
$$

Consequently, the fixed point inclusion $u \in N(u)$ is generalized Ulam-Hyers-Rassias stable. This completes the proof.

\section{An EXAMPLE}

Consider the class of differential inclusions with not instantaneous impulses of the form

$$
\left\{\begin{array}{lcc}
D_{0, t}^{r} z(t, x)-\frac{\partial^{2} z}{\partial x^{2}}(t, x) \in F(t, z(t, x)) ; & t \in[0,1] \cup(2,3], & x \in[0, \pi], \\
z(t, x)=g(t, z(t, x)) ; & t \in(1,2], & x \in[0, \pi], \\
z(t, 0)=z(t, \pi)=0 ; & t \in[0,1] \cup(2,3], & \\
z(0, x)=\phi(x) ; & x \in[0, \pi], &
\end{array}\right.
$$

where $D_{0, t}^{r}:=\frac{\partial^{r}}{\partial t^{r}}$ denotes the Caputo partial derivative of arbitrary order $r \in(0,1]$ with respect to $t$, i.e.,

$$
{ }^{c} D_{0, t}^{t} z(t, x)=\frac{1}{\Gamma(1-r)} \int_{0}^{t}(t-\tau)^{-r} \frac{\partial}{\partial \tau} z(\tau, x) d \tau,
$$


$F:([0,1] \cup(2,3]) \times \mathbb{R} \rightarrow \mathscr{P}_{c p}(\mathbb{R})$ is given by

$$
F(t, z(t, x))=\left\{v \in \mathbb{R}:\left|f_{1}(t, z(t, x))\right| \leq|v| \leq\left|f_{2}(t, z(t, x))\right|\right\} ;
$$

$t \in[0,1] \cup(2,3], x \in[0, \pi]$, where $f_{1}, f_{2}:([0,1] \cup(2,3]) \times \mathbb{R} \rightarrow \mathbb{R}$ such that

$$
f_{1}(t, z(t, x))=\frac{e^{t-3}}{111(1+|z(t, x)|)} ; t \in[0,1] \cup(2,3], x \in[0, \pi],
$$

and

$$
f_{2}(t, z(t, x))=\frac{e^{t-3}(1+z(t, x))}{111(1+|z(t, x)|)} ; t \in[0,1] \cup(2,3], x \in[0, \pi],
$$

$g:(1,2] \times \mathbb{R} \rightarrow \mathbb{R}$ is given by

$$
g(t, z(t, x))=\frac{1}{1+110 e^{t+x}} \arctan \left(t^{2}+|z(t, x)|\right) ; t \in(1,2], x \in[0, \pi],
$$

and $\phi:[0, \pi] \rightarrow \mathbb{R}$ is a continuous function. Hence the assumption $\left(H_{1}\right)$ is satisfied. Let $E=L^{2}([0, \pi], \mathbb{R})$ and define $B: D(B) \subset E \rightarrow E$ by $B w=w^{\prime \prime}$ with domain

$$
D(B)=\left\{w \in E: w, w^{\prime} \text { are absolutely continuous, } w^{\prime \prime} \in E, w(0)=w(\pi)=0\right\} .
$$

It is known that $B$ is the infinitesimal generator of an analytic semigroup on $E$ (see [34]). Then

$$
B w=-\sum_{n=1}^{\infty} n^{2}<w, e_{n}>e_{n} ; w \in D(A)
$$

where

$$
e_{n}(x)=\sqrt{\frac{2}{\pi}} \sin (n x) ; x \in[0, \pi], n=1,2, \ldots
$$

Clearly $B$ generates a compact semigroup $T(t) ; t>0$ given by

$$
T(t) w=\sum_{n=1}^{\infty} e^{-n^{2} t}<w, e_{n}>e_{n} ; w \in E
$$

For $x \in[0, \pi]$, set

$$
\begin{gathered}
u(t)(x)=z(t, x) ; \quad t \in[0,3], \\
u_{0}(x)=z(0, x)=\phi(x), \\
B u(t)(x)=\frac{\partial^{2} z}{\partial x^{2}}(t, x) ; \quad t \in[0,1] \cup(2,3], \\
F(t, u(t))(x)=F(t, z(t, x)) ; \quad t \in[0,1] \cup(2,3], \\
g(t, u(t))(x)=g(t, z(t, x)) ; \quad t \in(1,2] .
\end{gathered}
$$

Thus, under the above definitions of $\phi, A, F$ and $g$, the system (4.1) can be represented by the functional abstract problem (1.1), and solutions of (4.1) are solutions of the fixed point inclusion $u \in \Upsilon(u)$ where 
$\Upsilon: P C \rightarrow \mathscr{P}(P C)$ is the multivalued operator defined by

$$
(\Upsilon u)(t)=\left\{h \in P C:\left\{\begin{array}{l}
h(t)=S_{r}(t) u_{0} \\
+\int_{0}^{t}(t-\tau)^{r-1} T_{r}(t-\tau) f(\tau) d \tau ; t \in[0,1] \\
h(t)=g(t, u(t)) ; t \in(1,2] ; \\
h(t)=S_{r}(t-2) g_{k}(2, u(2)) \\
+\int_{2}^{t}(t-\tau)^{r-1} T_{r}(t-\tau) f(\tau) d \tau ; t \in S_{F \circ u}
\end{array}\right\}\right.
$$

For each $z, \bar{z}, \in E, t \in[0,1] \cup(2,3]$ and $x \in[0, \pi]$, we have

$$
|h(t, x)-\bar{h}(t, x)| \leq \frac{e^{t-3}}{111}|z(t, x)-\bar{z}(t, x)|,
$$

where $h \in S_{F \circ z}$ and $\bar{h} \in S_{F \circ \bar{z}}$. Thus we get

$$
\|h-\bar{h}\|_{E} \leq \frac{1}{111}\|z-\bar{z}\|_{E} .
$$

Therefore,

$$
H_{d}(F(t, z(t, x)), F(t, \bar{z}(t, x))) \leq \frac{1}{111}\|z-\bar{z}\|_{E} .
$$

Also, for each $z, \bar{z}, \in E, t \in(1,2]$ and $x \in[0, \pi]$, we can easily get

$$
\|g(t, z)-g(t, \bar{z})\|_{E} \leq \frac{1}{111}\|z-\bar{z}\|_{E} .
$$

Thus $\left(H_{2}\right)$ and $\left(H_{3}\right)$ are satisfied with $l_{F}=l_{g}=\frac{1}{111}$. It is easy seen that (3.1) is satisfied with $M=1$ and $a=3$. Indeed, for each $r \in(0,1]$, we get

$$
\begin{aligned}
\ell_{F} & =M l_{g}+\frac{M l_{F} a^{r}}{\Gamma(r)} \\
& =\frac{1}{111}+\frac{3^{r}}{111 \Gamma(r)} \\
& <\frac{7}{111}<1 .
\end{aligned}
$$

Hence, by Theorem 3.2, problem (4.1) has mild solutions, and $\Upsilon$ is a $\left(k_{\Upsilon}-M W P O\right)$ with $k_{\Upsilon}=\frac{1}{1-\ell_{F}}$. Moreover, $u \in \Upsilon(u)$ is stable in the sense of Ulam-Hyers. Also, hypothesis $\left(H_{4}\right)$ is satisfied with $\Phi(t)=t$ and $\lambda_{\Phi}=\frac{3^{r}}{\Gamma(2+r)}$. Indeed, for each $(t, x) \in[0,3] \times[0,1]$, we get

$$
\left(I_{0}^{r} \Phi\right)(t)=\frac{\Gamma(2) t^{1+r}}{\Gamma(2+r)} \leq \frac{3^{r} t}{\Gamma(2+r)}=\lambda_{\Phi} \Phi(t) .
$$

Consequently, $u \in \Upsilon(u)$ is stable in the sense of the generalized Ulam-Hyers-Rassias.

\section{CONCLUSION}

In this paper, we provided sufficient conditions for the existence of mild solutions and the Ulam stability of these solutions for a class of semilinear fractional differential inclusions with not instantaneous impulses in separable Banach spaces. The notion of Picard operators and the semigroup theory are the main tools for the proof of the main results. An illustrative example was also presented. 


\section{REFERENCES}

[1] S. Abbas, M. Benchohra, Fractional order partial hyperbolic differential equations involving Caputo's derivative, Stud. Univ. Babeş-Bolyai Math. 57 (2012), 469-479.

[2] S. Abbas, M. Benchohra, Ulam-Hyers stability for the Darboux problem for partial fractional differential and Integrodifferential equations via Picard operators, Results Math. 65 (2014), 67-79.

[3] S. Abbas, M. Benchohra, J.J. Nieto, Ulam stabilities for impulsive partial fractional differential equations, Acta Univ. Palack. Olomuc. Fac. Rerum Natur. Math. 53 (2014), 5-17.

[4] K. Diethelm, N. J. Ford, Analysis of fractional differential equations, J. Math. Anal. Appl. 265 (2002), 229-248.

[5] L. Wang, B. Zhang, Infinitely many solutions for Schrodinger-Kirchhoff type equations involving the fractional $p$ Laplacian and critical exponent, Electron. J. Differential Equations 2016 (2016), Article ID 339.

[6] M. Xiang, B. Zhang, Z. Wei, Existence of solutions to a class of quasilinear Schrodinger systems involving the fractional p-Laplacian, Electron. J. Qual. Theory Differ. Equ. 2016 (2016), Article ID 107.

[7] I. A. Rus, Fixed points, upper and lower fixed points: abstract Gronwall lemmas, Carpathian J. Math. 20 (2004), $125-134$.

[8] I. A. Rus, Picard operators and applications, Sci. Math. Japonicae 58 (2003), 191-219.

[9] I. A. Rus, Generalized Contractions and Applications, Cluj University Press, Cluj- Napoca, 2001.

[10] I. A. Rus, Remarks on Ulam stability of the operatorial equations, Fixed Point Theory 10 (2009), 305-320.

[11] E. Hernández, D. O’Regan, On a new class of abstract impulsive differential equations, Proc. Amer. Math. Soc. 141 (2013), 1641-1649.

[12] M. Pierri, D. O'Regan, V. Rolnik, Existence of solutions for semi-linear abstract differential equations with not instantaneous, Appl. Math. Comput. 219 (2013), 6743-6749.

[13] I. A. Rus, Ulam stabilities of ordinary differential equations in a Banach space, Carpathian J. Math. 26 (2010), 103-107.

[14] J. Wang, M. Fečkan, Y. Zhou, Ulam's type stability of impulsive ordinary differential equations, J. Math. Anal. Appl. 395 (2012), 258-264.

[15] J. Wang, Y. Zhang, Existence and stability of solutions to nonlinear impulsive differential equations in $\beta$-normed spaces, Electron. J. Differential Equations 2014 (2014), Article ID 83.

[16] S. G. Samko, A. A. Kilbas, O. I. Marichev, Fractional Integrals and Derivatives: Theory and Applications, Gordon and Breach, Yverdon, 1993.

[17] K. Deimling, Multivalued Differential Equations, Walter De Gruyter, Berlin-New York, 1992.

[18] L. Gorniewicz, Topological Fixed Point Theory of Multivalued Mappings, Mathematics and its Applications, 495, Kluwer Academic Publishers, Dordrecht, 1999.

[19] Sh. Hu, N. Papageorgiou, Handbook of Multivalued Analysis, Volume I: Theory, Kluwer Academic Publishers, Dordrecht, 1997.

[20] M. Kisielewicz, Differential Inclusions and Optimal Control, Kluwer Academic Publishers, Dordrecht, The Netherlands, 1991.

[21] K. Yosida, Functional Analysis, $6^{\text {th }}$ ed. Springer-Verlag, Berlin, 1980.

[22] I. A. Rus, Weakly Picard operators and applications, Seminar on Fixed Point Theory, Cluj-Napoca 2 (2001), 41-58.

[23] A. Petruşel, Multivalued weakly Picard operators and applications, Sci. Math. Japon. 59 (2004), 167-202.

[24] I. A. Rus, A. Petrusel, A. Sîtămărian, Data dependence of the fixed points set of some multivalued weakly Picard operators, Nonlinear Anal. 52 (2003), 1947-1959.

[25] H. Covitz, S. B. Nadler Jr., Multivalued contraction mappings in generalized metric spaces, Israel J. Math. 8 (1970), 5-11.

[26] M. M. El-Borai, Some probability densities and fundamental solutions of fractional evolution equations, Choas, Solitons Fractals 14 (2002), 433-440.

[27] Y. Zhou, F. Jiao, Existence of mild solutions for fractional netural evolution equations, Comput. Math. Appl. 59 (2010), 1063-1077.

[28] T.P. Petru, A. Petrusel. J.-C. Yao, Ulam-Hyers stability for operatorial equations and inclusions via nonself operators, Taiwanese J. Math. 15 (2011), 2169-2193.

[29] L. Guran, Fixed points for multivalued contractions on a metric space, Surveys Math. Appl. 5 (2010), 191-199.

[30] J.-P. Aubin, H. Frankowska, Set-Valued Analysis, Birkhauser, Basel, 1990. 
[31] D. Henry, Geometric theory of Semilinear Parabolic Partial Differential Equations, Springer-Verlag, Berlin-New York, 1989.

[32] C. Castaing, M. Valadier, Convex Analysis and Measurable Multifunctions, Lecture Notes in Mathematics, 580, SpringerVerlag, Berlin-Heidelberg-New York, 1977.

[33] L. Rybinski, On Carathédory type selections, Fund. Math. 125 (1985), 187-193.

[34] A. Pazy, Semigroups of Linear Operators and Applications to Partial Differential Equations, Springer-Verlag, New York, 1983. 